\title{
Gamma Knife radiosurgery combined with stereotactic aspiration as an effective treatment method for large cystic brain metastases
}

\author{
HONGWEI WANG ${ }^{1}$, SONGTAO QI $^{1}$, CHANGWU DOU $^{2}$, HAITAO JU $^{2}$, ZHANBIAO HE $^{2}$ and QINGHAI MA ${ }^{2}$ \\ ${ }^{1}$ Department of Neurosurgery, Nanfang Hospital Southern Medical University, Guangzhou, Guangdong 510515; \\ ${ }^{2}$ Department of Neurosurgery, Affiliated Hospital of Inner Mongolia Medical College, \\ Hohhot, Inner Mongolia 010050, P.R. China
}

Received January 26, 2015; Accepted April 1, 2016

DOI: $10.3892 / \mathrm{ol} .2016 .4603$

\begin{abstract}
In the present study, the efficacy and clinical outcomes of stereotactic aspiration combined with the Gamma Knife radiosurgery (GKRS) method were evaluated retrospectively for patients with large cystic brain metastases. This combined method aims to decrease the tumor weight (volume) and increase the possible radiation dose. The present study involved 48 patients who were diagnosed with cystic metastatic brain tumors between January 2008 and December 2012 in the Department of Neurosurgery of Nanfang Hospital Southern Medical University (Guangzhou, China). Every patient underwent Leksell stereotactic frame, 1.5T magnetic resonance imaging (MRI)-guided stereotactic cyst aspiration and Leksell GKRS. Subsequent to the therapy, MRI was performed every 3 months. The results indicated that 48 cases were followed up for 24-72 months, with a mean follow-up duration of 36.2 months. Following treatment, 44 patients $(91.7 \%)$ exhibited tumor control and 4 patients $(8.3 \%)$ experienced progression of the local tumor. During this period, 35 patients $(72.9 \%)$ succumbed, but only $2(4.2 \%)$ of these succumbed to the brain metastases. The total local control rate was $91.7 \%$ and the median overall survival time of all patients was 19.5 months. The 1-year overall survival rate was $70.8 \%$ and the 2-year overall survival rate was $26.2 \%$. In conclusion, these results indicated that the method of stereotactic cyst aspiration combined with GKRS was safe and effective for patients with large cystic brain metastases. This method is effective for patients whose condition is too weak for general anesthesia and in whom the tumors are positioned at eloquent areas. This method enables patients to
\end{abstract}

Correspondence to: Professor Songtao Qi, Department of Neurosurgery, Nanfang Hospital Southern Medical University, 1838 Guangzhou Road North, Guangzhou, Guangdong 510515, P.R. China

E-mail: qisongtgz@yeah.net

Key words: stereotactic cyst aspiration, Gamma Knife radiosurgery, cystic brain metastases, colloidal phosphorus-32 avoid a craniotomy, and provides a good tumor control rate, survival time and quality of life.

\section{Introduction}

In the clinical setting, metastatic tumors that translocate from the primary tumor site to the central nervous system are the most frequently occurring intracranial tumors (1). Three methods, including stereotactic radiosurgery, general surgery and whole-brain radiation therapy (WBRT), are normally clinically used and are relatively effective for treating brain metastases (1). However, for a long time, none of these methods was assigned as the standard therapy in clinical practice. The median survival time of affected patients is 1-3 months without therapy (1). The median survival time increases to $\sim 6$ months after WBRT (2) and to 10 months subsequent to using the Gamma Knife radiosurgery (GKRS) method (3). The survival time has been recorded as 12 months in patients who underwent surgery (prior method) and WBRT (4). With each of the latter methods, the patients only suffered from single brain metastasis, however, when using the GKRS and WBRT combined method for multiple brain metastases, a survival time of $>1$ year can also be obtained (5). Recently, the radiosurgery method has become a focal topic in the area of brain tumor metastases, and more advantages have been demonstrated when compared to tumor resection. Radiosurgery has been applied in multiple metastatic lesions and in metastases in deep locations, with satisfactory outcomes (6). Radiosurgery can also be used for patients with other major medical issues considered as contraindications for general anesthesia and surgery (6). In particular, radiosurgery and stereotactic surgery can be combined to complete treatment; as these two treatment methods are based on the same technology platform, a number of the patients with cystic metastases can avoid craniotomy.

Due to cystic components, a number of brain metastases are characterized by a large volume. The clearance of this cystic component is critical for the surgical removal of the tumor. However, certain factors could affect the effects of the surgery, including tumor multiplicity, patient functional status and lesion location. Due to the large volume of the tumor, the radiosurgery method is not suitable for cystic metastatic tumors. In order to treat such lesions using the 
radiosurgery method, it is critical to decrease the cystic component volume. Stereotactic cyst aspiration, a minimally invasive surgical approach can overcome these shortcomings, and is not affected by the location of the lesion, the number of lesions or the patient status (6). Following stereotactic aspiration of the cystic component, the present study evaluated the clinical outcomes and efficacy of the GKRS method for the treatment of cystic brain metastases by assessing the tumor volume.

\section{Materials and methods}

Patients. A total of 48 patients diagnosed with large cystic brain metastases between January 2008 and December 2012 were involved in this study of GKRS. All patients were diagnosed with single large cystic brain metastases. Furthermore, the primary malignancy was determined for each patient, and no patient had a malignancy of unknown primary site. The pathological results are listed in Table I. Samples were obtained as previously described (6). Consequently, the patients also underwent magnetic resonance imaging (MRI) to diagnose the brain metastases. The present study was approved by the Ethics Committee of the Southern Medical University (Guangzhou, China). Informed consent was obtained from all patients.

The 48 patients included 28 males and 20 females, with a mean age of 61.4 years old (range, 33-78 years). In this study, the primary tumors consisted of small cell lung cancer (6 patients, $12.5 \%$ ), non-small cell lung cancer (29 patients, $60.4 \%$ ), breast cancer (5 patients, $10.4 \%$ ), esophageal cancer (2 patients, $4.2 \%$ ), colon and rectal cancer (4 patients, $8.3 \%$ ), and kidney cancer (1 patients, 2.1\%). The initial symptoms of brain metastasis included headaches (25 patients, 52.1\%), seizures (1 patient, 2.1\%), motor weakness (14 patients, $29.2 \%$ ), visual disturbance (2 patients, $4.2 \%$ ) and gait disturbance (6 patients, $12.5 \%$ ). The average number of brain metastases was 2.4 (range, 1-7). The mean Karnofsky performance status (KPS) score was 71.3 (range, 50-90) (5). The basic data and characteristics of the patients are listed in Table I.

MRI. After positioning the stereotactic frame (Leksell G frame; Elekta AB, Stockholm, Sweden), MRI was performed using the vantage Titan 3T MRI machine (Toshiba, Japan). The MR T1-weighted images with gadolinium contrast were recorded and analyzed. Meanwhile, the slices were also reconstructed in the axial plane every $3 \mathrm{~mm}$. The Leksell SurgiPlan system (Elekta AB) was applied to regulate the surgical trajectory. The stereotactic aspiration of the large cystic brain metastases was performed and recorded in the operating room. The findings showed that the mean tumor volume was $26.8 \mathrm{cc}$ (range, 19.0-75.7 cc) and the aspiration fluid volume was $\sim 80 \%$ of the cyst volume. For the prevention of tumor hemorrhages, 18 lesions (37.5\%) with a tumor volume $>40 \mathrm{ml}$ were treated with drainage tube insertion for $24 \mathrm{~h}$ following the stereotactic aspiration. It was confirmed that all of the patients presented with a markedly reduced cyst volume after the stereotactic drainage.

A second set of MR images was recorded after cystic drainage. At the same time, the drainage tube was closed until
Table I. Basic data and clinical characteristics of the 48 study patients.

\begin{tabular}{lc}
\hline Characteristics & Patients, $\mathrm{n}(\%)$ \\
\hline Age, years & \\
$<60$ & $15(31.3)$ \\
$>60$ & $33(68.8)$ \\
Gender & \\
Male & $28(58.3)$ \\
Female & $20(41.7)$ \\
Initial symptom & \\
Motor weakness & $25(52.1)$ \\
Headaches & $14(29.2)$ \\
Gait disturbance & $6(12.5)$ \\
Visual disturbance & $2(4.2)$ \\
Seizure & $1(2.1)$ \\
Primary tumor & \\
Non-small cell lung cancer & $29(60.4)$ \\
Small cell lung cancer & $6(12.5)$ \\
Breast cancer & $5(10.4)$ \\
Colon and rectal cancer & $4(8.3)$ \\
Esophageal cancer & $2(4.2)$ \\
Kidney cancer & $1(2.1)$ \\
Malignant melanoma & $1(2.1)$ \\
Number of metastases & \\
1 & $18(37.5)$ \\
2 & $10(20.8)$ \\
3 & $13(27.1)$ \\
4 & $7(14.6)$ \\
KPS score & \\
50 & $3(6.3)$ \\
60 & $8(16.7)$ \\
70 & $21(43.8)$ \\
80 & $12(25.0)$ \\
90 & $4(8.3)$ \\
\hline &
\end{tabular}

KPS, Karnofsky performance status.

the end of Gamma Knife treatment. The Leksell GammaPlan system (Elekta AB) was used to plan the GKRS. For the GKRS method, the mean tumor volume was decreased from $26.8 \mathrm{cc}$ (range, 19.0-75.7 cc) to $5.4 \mathrm{cc}$ (range, 1.0-16.0 cc). The average prescribed radiation dose to the tumor margin was 18 Gy (range, 14-20 Gy). Ten cases with a tumor volume of $<40 \mathrm{cc}$ underwent exposure to $18 \mathrm{~Gy}$ for $20 \mathrm{~min}$. The other 8 patients underwent exposure to $16 \mathrm{~Gy}$ for 20 min Prior to pulling out the drainage tube, the patients were administered supplement colloidal phosphorus-32 radiation therapy $(0.01 \mathrm{mCi} / \mathrm{ml})$. This method is often used in the treatment of cystic craniopharyngioma (7). In this study, the GKRS was also applied to other areas of the brain, in which, however, no the large cysts were observed.

MRI was performed again after an interval of 3 months (Figs. 1 and 2). Tumor control was represented as a decrease 

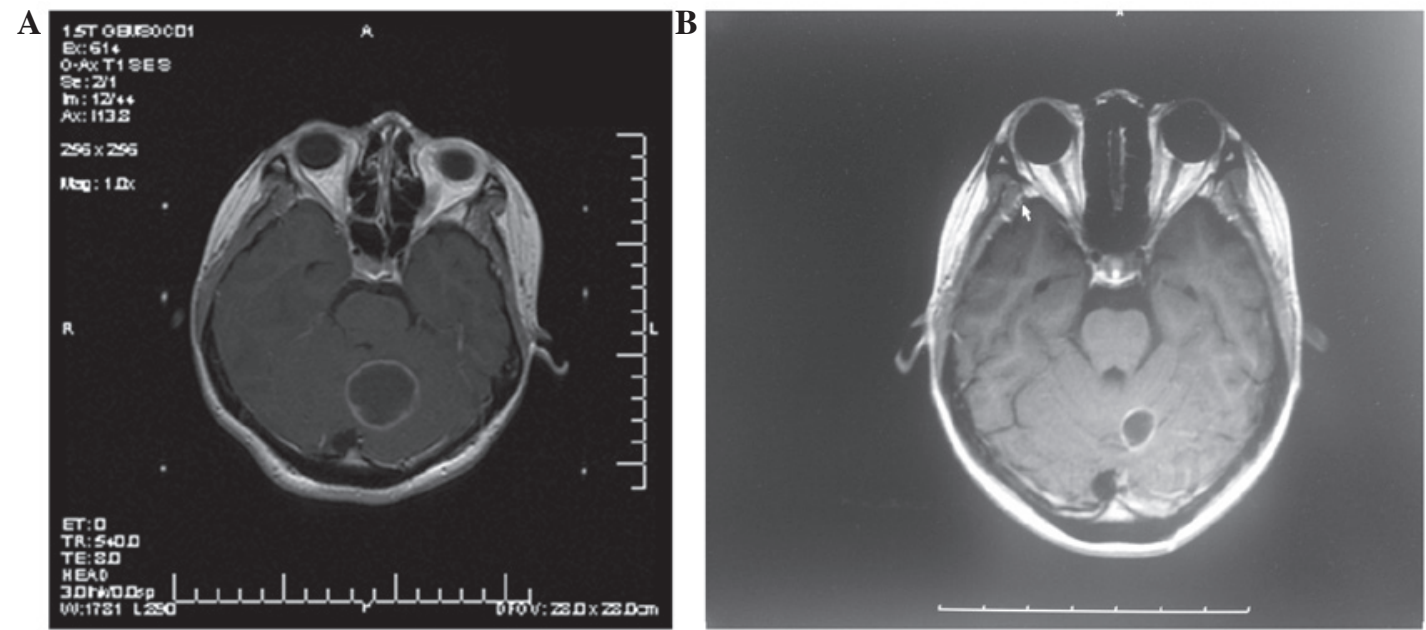

Figure 1. T1-weighted (axial contrast-enhanced) magnetic resonance imaging of a 24-year-old woman diagnosed with a large cystic brain metastasis. The prescribed dose for the Gamma Knife radiosurgery (GKRS) was $16 \mathrm{~Gy}$, with 0.5 mci colloidal phosphorus-32 radiation therapy. (A) Prior to aspiration. (B) Subsequent to aspiration at 3 months post-GKRS.
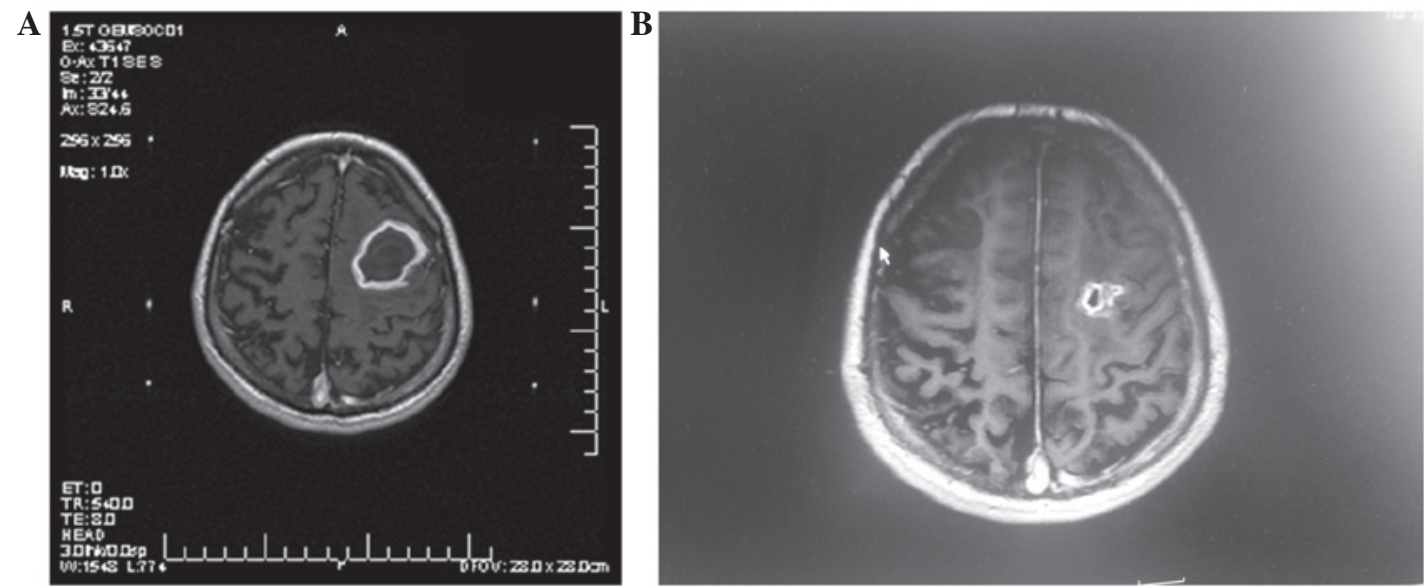

Figure 2. T1-weighted (axial contrast-enhanced) magnetic resonance imaging of a 45-year-old man diagnosed with a large cystic brain metastasis. The prescribed dose of Gamma Knife radiosurgery (GKRS) was $18 \mathrm{~Gy}$. (A) Prior to aspiration. (B) Subsequent to aspiration at 3 months post-GKRS.

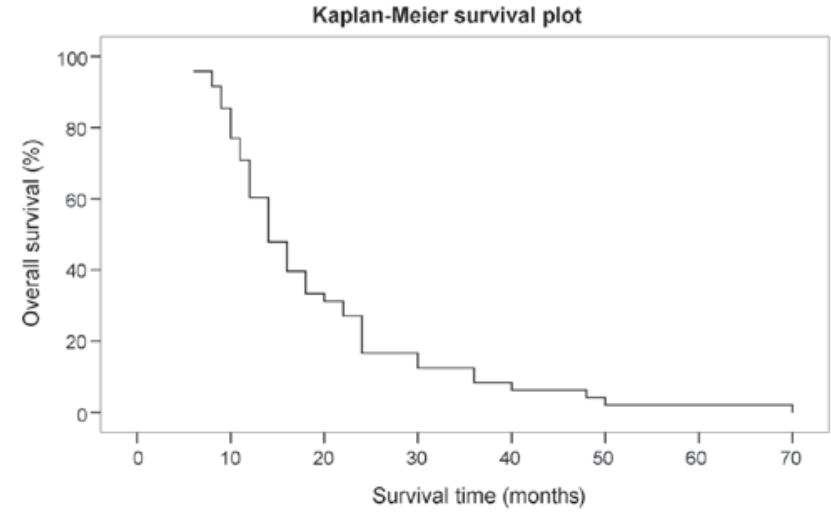

Figure 3. Kaplan-Meier plot showing the overall survival rate of the patients.

in the tumor size following treatment. Progression of the local tumor was represented as an increase in size of the previously treated tumor. Moreover, the progression of the remote tumor was represented as the appearance of new brain metastases.

\section{Results}

Subsequent to surgery, there was no bleeding, infection complications or surgical mortality. All the post-operative clinical symptoms showed improvement at 1 week post-surgery and the mean KPS score was 90. MRI enhanced scanning of the head after 3 months showed tumor control, with tumor shrinkage or disappearance, and significant reduction of the edema around the tumor in 44 cases $(91.7 \%)$, while local tumor progression, with no radioactive brain necrosis, occurred in 4 cases $(8.3 \%)$. The 10 patients who underwent phosphorus-32 radiotherapy also exhibited no radioactive brain edema. Of the 8 cases $(16.7 \%)$ that exhibited remote tumor progression, $4(8.3 \%)$ received WBRT and 3 cases $(6.3 \%)$ received chemotherapy. The mean follow-up time of the patients was 36.2 months (range, 24-72 months). A total of 12 cases $(25.0 \%)$ remained alive and 36 patients (75.0\%) succumbed during the follow-up period. Whereas only 4 patients $(8.3 \%)$ succumbed to brain metastasis progression, 2 patients (4.2\%) succumbed due to unrelated other diseases and 30 patients $(62.5 \%)$ succumbed due to 
primary cancer progression. The overall median survival time of the patients following the GKRS treatment was 19.5 months (range, 6-70 months), while the 1-year survival rate was $70.8 \%$ and the 2-year survival rate was $26.2 \%$ (Fig. 3). Furthermore, the mean KPS score of the patients was 97.7 (range, 80-100).

\section{Discussion}

Metastatic brain tumors are normally composed of cystic components, however, the reasons for the cyst formation have not been clearly investigated (8). Stem (9) reported that the brain cyst fluid protein always presents in the inflammatory exudates. Cumings (10) also reported that the cyst fluid formation may be correlated with the tumor degeneration. Gardner et al (11) found that fluid accumulating in brain tumors runs in the normal drainage route, since there are no lymphatic vessels in the tumors. Conventionally, in the clinic, the presence of a large, single, cystic brain tumor is considered as an effective indication for surgery (6). A study by Yoshida and Morii (12) reported rapid neurological symptom relief for these large cystic lesions after surgery. However, surgery cannot treat deep lesions within the brain, and may cause severe neurologic outcomes. Moreover, surgical procedures are not safe for patient's with a poor general condition, as well as being ineffective for those individuals with multiple lesions. More recently, the stereotactic radiosurgical procedure and the GKRS procedure have been combined and applied to cerebral metastatic disease (13). This method has proved to be valuable due to its minimal invasiveness, which leads to a decreased hospitalization time and lower hospital cost. This method could also result in a low associated tumor morbidity and an excellent local tumor control rate $(14,15)$. The results for the GKRS method in tumor patients with multiple or solitary lesions have been reported to be an improvement on those of other previously reported methods (16).

Radiosurgery is not always applicable for large cystic brain metastases. Pan et al (17) reported that GKRS alone could not control the large cystic components $(>10 \mathrm{ml})$ effectively. When applied to the solid component of the tumor, the combination of stereotactic cyst aspiration and GKRS resulted in a good outcome, with a 1-year survival rate of $60 \%$. A study by Franzin et al (6) also indicated that $~ 39.1 \%$ patients did not experience remote or local tumor progression, that $26.3 \%$ of patients succumbed from neurological progression and that the overall median survival was 15 months when stereotactic drainage and GKRS were applied for the treatment of cystic brain metastases. In the present study, $91.7 \%$ of patients showed marked tumor inhibition, the overall median survival time was 19.5 months and only $8.3 \%$ patients succumbed from brain metastasis progression post-treatment. We hypothesize that this result may be associated with the higher dose exposure to the tumor; phosphorus-32 compensated for the inadequacy of the Gamma Knife exposure dose for the larger lesions. Following the resection of single brain metastases, Tendulkar et al (18) reported that the median patient survival time was 10.6 months. Hong et al (19) found that when surgical resection was performed in NSCLC patients with multiple brain metastases, the median survival time was 10.8 months. Faguer et al (20) reported the use of radiosurgery delivered to the tumor bed for 22 patients with metastases. The overall median survival time of the patients was 13 months compared with 11 months for the patients who received WBRT; however, this difference was not significant.

It is difficult to completely remove tumors if they are distributed in deep locations or eloquent areas, or if they have a large cystic volume (5). In these situations, the GKRS method combined with stereotactic cyst aspiration could be a better modality compared with surgical resection, as a number of patients are too weak to undergo general anesthesia. Such findings further confirm the efficacy of the GKRS method when combined with stereotactic cyst aspiration for the treatment of unresectable cystic brain metastases in the clinic.

There are certain complications associated with brain tumors, including seizures, neurosurgical deficits, hemorrhage and infection. However, the mortality rate of patients in certain large series has been recorded at $<1 \%$, and the complication rates even increase from 0 to $7 \%$ following specific treatments (21). The complications of stereotactic radiosurgery are always associated with the proximity of structures and the radiation to the brain (5). There are also a number of associated early complications, including worsening neurological deficits and seizures, however, these are rare (6). A few patients also exhibit mild transitory symptoms, such as nausea, headaches and dizziness. Complications in the late stage (6 to 9 months after the procedure) may also be observed, including trigeminal neuropathy, facial palsy and visual symptoms $(5,6)$. However, Franzin et al (6) found that no acute complications were observed and that only $7.6 \%$ of patients underwent radionecrosis following treatment with GKRS combined with stereotactic aspiration for cystic brain metastases. Jung et al (22) reported that stereotactic cyst aspiration and radiosurgery for cystic brain metastases resulted in a local control rate of 58.6\%; this type of treatment is safe, but the efficiency is not high. In the present study, 10 cases of phosphorus-32 radiotherapy patients without radioactive brain necrosis were also recorded. The main treatment was largely composed of two stereotactic procedures, consisting of stereotactic cyst aspiration and GKRS, which is performed by using a single frame application (23). Therefore, stereotactic cyst aspiration combined with GKRS could reduce the hospitalization time and cost, and cause less inconvenience to the patients.

In conclusion, the cyst aspiration method can decrease the associated risks of radiation necrosis and complications, and decrease the tumor volume. The present data support the application and safety of the combined stereotactic cyst aspiration and GKRS method, which was effective for patients with large, single, cystic brain metastases. The method is particularly useful for patients whose overall condition is poor, which typically inhibits the use of general anesthesia. Furthermore, patients with deeply located metastatic lesions or multiple lesions could also benefit from this treatment.

\section{References}

1. Sun X, Chen Z, Yang W, Yu F, Zhao J, He P and Wang Z: Rare incidence of a diffuse brain metastatic carcinoma: A case report. Oncol Lett 8: 1807-1809, 2014.

2. Diener-West M, Dobbins TW, Phillips TL and Nelson DF: Identification of an optimal subgroup for treatment evaluation of patients with brain metastases using RTGO study 7916. Int J Radiat Oncol Bio Phys 16: 669-673, 1989. 
3. Andrews DW, Scott CB, Sperduto PW, Flanders AE, Gaspar LE, Schell MC, Werner-Wasik M, Demas W, Ryu J, Bahary JP, et al: Whole brain radiation therapy with or without stereotactic radiosurgery boost for patients with one to three brain metastases: Phase III results of the RTGO 9508 randomized trial. Lancet 363 : 1665-1672, 2004.

4. Tsao MN, Lioyd N, Wong R, Chow E, Rakovitch E and Laperriere N: Whole brain radiotherapy for the treatment of multiple brain metastases. Cochrane Database Syst Rev 4 CD003869, 2012.

5. Aoyama H, Shirato H, Tago M, Nakagawa K, Toyoda $T$, Hatano K, Kenjyo M, Oya N, Hirota S, Shioura H, et al: Stereotactic radiosurgery plus whole-brain radiation therapy vs. stereotactic radiosurgery alone for treatment of brain metastases: A randomized controlled trial. JAMA 295 2483-2491, 2006

6. Franzin A, Vimercati A, Picozzi P, Serra C, Snider S, Gioia L, Ferrari da Passano C, Bolognesi A and Giovanelli M: Stereotactic drainage and gamma knife radiosurgery of cystic brain metastasis. J Neurosurg 109: 259-267, 2008.

7. Barriger RB, Chang A, Lo SS, Timmerman RD, DesRosiers C, Boaz JC and Fakiris AJ: Phosphorus-32 therapy for cystic craniopharyngiomas. Radiother Oncol 98: 207-212, 2011.

8. Kim MS, Lee SL and Sim SH: Brain tumors with cyst treated with gamma knife radiosurgery: Is microsurgery indicated? Stereotact Funct Neurosurg 72 (Suppl 1): S38-S44, 1999.

9. Stem K: Chemical study of fluids obtained from cerebral cysts: Report on 56 cases. Brain 62: 88, 1939.

10. Cumings JN: The chemistry of cerebral cysts. Brain 73: 244-250, 1950.

11. Gardner WJ, Collis JS Jr and Lewis LA: Cystic brain tumors and the blood-brain barrier. Comparison of protein fractions in cyst fluids and sera. Arch Neurol 8: 291-298, 1963.

12. Yoshida S and Morii K: The role of surgery in the treatment of brain metastasis: A retrospective review. Acta Neurochir (Wien) 146: 767-770, 2004.
13. Higuchi F, Kawamoto S, Abe Y, Kim P, Ueki K: Effectiveness of a 1-day aspiration plus gamma knife surgery procedure for metastatic brain tumor with a cystic component. J Neurosurg 117 (Suppl): S17-S22, 2012.

14. Uchino M, Nagao T, Seiki Y, Shibata I, Terao H and Kaneko I: Radiosurgery for cystic metastatic brain tumor. No Shinkei Geka 28: 417-421, 2000 (In Japanese).

15. Mingione V, Oliveira M, Prasar D, Steiner M and Steiner L: Gamma surgery for melanoma metastases in the brain. J Neurosurg 96: 544-551, 2002.

16. Flickiner JC: Radiotherapy and radiosurgical management of brain metastases. Curr Oncol Rep 3: 484-489, 2001.

17. Pan HC, Sheehan J, Stroila M, Steiner M and Steiner L: Gamma knife radiosurgery for brain metastases from lung cancer. J Neurosurg 102 (Suppl): S128-S133, 2005.

18. Tendulkar RD, Liu SW, Barnett GH, Vogelbaum MA, Toms SA, Jin T and Suh JH: RPA classification has prognostic significance for surgically resected single brain metastasis. Int J Radiat Oncol Biol Phys 66: 810-817, 2006.

19. Hong N, Yoo H, Gwak HS, Shin SH and Lee SH: Outcome of surgical resection of symptomatic cerebral lesions in non-small cell lung cancer patients with multiple brain metastases. Brain Tumor Res Treat 1: 64-70, 2013.

20. Faguer R, Boissonneau S, Menei P and Metellus P: Gamma knife radiosurgery to the tumor bed after resection of brain metastases. Neurochirurgie 60: 355, 2014.

21. Bernstein M and Parrent A: Complication of CT-guided stereotactic biopsy of intra-axial brain lesions. J Neurosurg 81: 165-168, 1994.

22. Jung TY, Kim IY, Jung S, Jang WY, Moon KS, Park SJ and Lim SH: Alternative treatment of stereotactic cyst aspiration and radiosurgery for cystic brain metastases. Stereotact Funct Neurosurg 92: 234-241, 2014.

23. Shen G, Wang YJ, Shen WJ, Zhou ZS, Wang JL, Sheng HG, Dong DP, Zhou M, Yang G, Wang QW and Zeng Y: Stereotactic body radiation therapy for centrally-located lung tumors. Oncol Lett 7: 1292-1296, 2014. 